\title{
DYNAMIC FRACTURE OF CONCRETE COMPACT TENSION SPECIMEN: MESH SENSITIVITY STUDY
}

\author{
D. RUTA* AND J. OŽBOLT ${ }^{\dagger}$ \\ ${ }^{*}$ Institute of Construction Materials (IWB), University of Stuttgart \\ Pfaffenwaldring 4, 70569 Stuttgart, Germany \\ e-mail: ozbolt@iwb.uni-stuttgart.de \\ e-mail: daniela.ruta@iwb.uni-stuttgart.de
}

Key words: CT Specimen, Dynamic Fracture, Microplane Model, Crack Band Approach.

\begin{abstract}
It is well known that without a proper regularization scheme the finite element analysis of softening materials leads to mesh dependent response. The results of the analysis should be as less as possible dependent on the choice of the finite element type and on the finite element discretization. In the present article the mesh sensitivity study of the compact tension specimen loaded under quasi-static and dynamic loading conditions is carried out. It is shown that relatively simple regularization scheme based on the element size independent energy dissipation (crack band method) leads to mesh insensitive results. In the analysis the constitutive law based on the rate sensitive microplane model for concrete is used. In the model the tensile response is based on the bi-linear crack-opening softening law. It is shown that, in spite of the simple regularization scheme, the rate dependent peak load, failure mode, crack branching and crack velocity are realistically predicted for different types and sizes of finite elements.
\end{abstract}

\section{INTRODUCTION}

To realistically simulate the behavior of concrete structures under dynamic loading, the numerical finite element (FE) analysis should account for the influence of high strain-rates. Moreover, the phenomena that are related with the hardening and softening of the material and the crack propagation (inertia effects) should also be properly accounted for. It is well known that without a proper regularization scheme the local $\mathrm{FE}$ analysis of softening materials leads to mesh dependent response. The results of the analysis should be as less as possible dependent on the choice of the finite element type and on the discretization.

There are principally two approaches to avoid spurious mesh sensitivity: (i) Crack band method that is based on the energy dissipation, which should be independent of the size of the finite elements and (ii) Nonlocal approaches or higher order continua. For concrete like materials the relatively simple crack band approach works fine for most cases. This is especially true for mode-I fracture, however, for combined compression-shear failure the analysis is objective only if the element size is in the range of the maximum aggregate size. This can be the limitation of the method, especially if detailed analysis of the problems is required, e.g. modeling of interface zone between reinforcement and concrete. The second groups of regularization strategies are more general, however there is a number of disadvantages such as high computational costs, problem with boundaries, calibration of macroscopic material properties and other.

The main aim of the present contribution is to study the objectivity of the regularization scheme based on the relatively simple energy approach (crack band method) employed in 
dynamic finite element fracture analysis of compact tension (CT) specimen. The scope is to investigate whether the approach is able to realistically account for dynamic fracture of concrete. In the first part of the paper a short description of the employed microplane constitutive law with bi-linear tension softening is given. Subsequently the mesh sensitivity study is performed on the CT specimen and conclusions are drawn out.

\section{MICROPLANE MODEL}

In the microplane model the material response is computed based on the monitoring of stresses and strains in different predefined directions. Integrating microplane stresses in a thermodynamically consistent way it is possible to calculate macroscopic stress tensor. In the model, material is characterized by the uni-axial relations between stress and strain components on planes of various orientations. Each microplane is defined by its unit normal vector $n_{i}$ (Fig. 1).
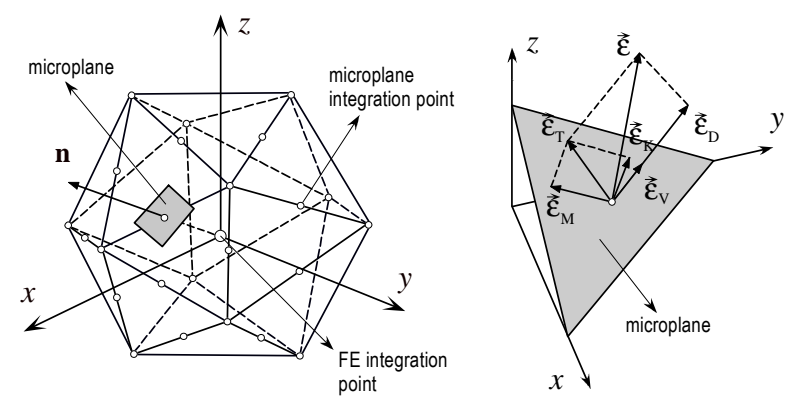

Figure 1: Decomposition of the macroscopic strain vector into microplane strain components - normal (volumetric and deviatoric) and shear linear tensile softening.

Microplane strains are assumed to be the projections of macroscopic strain tensor $\varepsilon_{i j}$ (kinematic constraint). On the microplane are considered normal $\left(\sigma_{\mathrm{N}}, \varepsilon_{\mathrm{N}}\right)$ and two shear stress-strain components $\left(\sigma_{\mathrm{M}}, \sigma_{\mathrm{K}}, \varepsilon_{\mathrm{M}}, \varepsilon_{\mathrm{K}}\right)$. To realistically model concrete, the normal microplane stress and strain components have to be decomposed into volumetric and deviatoric parts $\left(\sigma_{\mathrm{N}}=\sigma_{\mathrm{V}}+\sigma_{\mathrm{D}}, \quad \varepsilon_{\mathrm{N}}=\varepsilon_{\mathrm{V}}+\varepsilon_{\mathrm{D}}\right)$. Unlike to most microplane formulations for concrete, which are based on the kinematic constraint approach, to prevent unrealistic model response for dominant tension (strong localization), kinematic constraint is relaxed through discontinuity function $\psi$ [1]. In the originally proposed model this is an exponential function, which applies for all microplane components only if maximum principal stress $\sigma_{1}$ and volumetric strain $\varepsilon_{\mathrm{v}}$ are positive (tension). Recently, a new discontinuity function is introduced, which is based on linear or bi-linear tension softening. The function $\psi$ depends on maximum principal stress and volumetric strain. It applies only for dominant tension, i.e. $\sigma_{1}>0$ and $\varepsilon_{\mathrm{v}}>0$, otherwise $\psi=1.0$.

From the total microplane strain $\varepsilon_{\mathrm{m}}$, which is obtained based on the kinematic constraint from the total strain tensor $\varepsilon_{i j}$, the effective microplane strain $\varepsilon_{\mathrm{m}, \text { ef }}$ and microplane stress $\sigma_{\mathrm{m}}$ are computed as:

$$
\varepsilon_{\mathrm{m}, \mathrm{ef}}=\psi \varepsilon_{\mathrm{m}} \quad \text { and } \quad \sigma_{\mathrm{m}}=C_{\mathrm{m}, 0} \varepsilon_{\mathrm{m}, \mathrm{ef}}
$$

where $C_{\mathrm{m}, 0}$ is the elastic stiffness of the microplane component. Note that subscript ' $\mathrm{m}$ ' stays for the corresponding microplane component ( $\mathrm{V}, \mathrm{D}, \mathrm{M}$ and $\mathrm{K})$. For $\varepsilon_{1} \leq f_{\mathrm{t}} / E_{\mathrm{c}}$, $\psi=1.0$, i.e. the response is linear elastic. However, for $f_{\mathrm{t}} / E_{\mathrm{c}}<\varepsilon_{1} \leq \varepsilon_{\mathrm{cr}}, \psi$ is chosen such that the macroscopic tensile response follows linear or bi-linear crack-opening (softening) response of concrete (see Fig. 2).

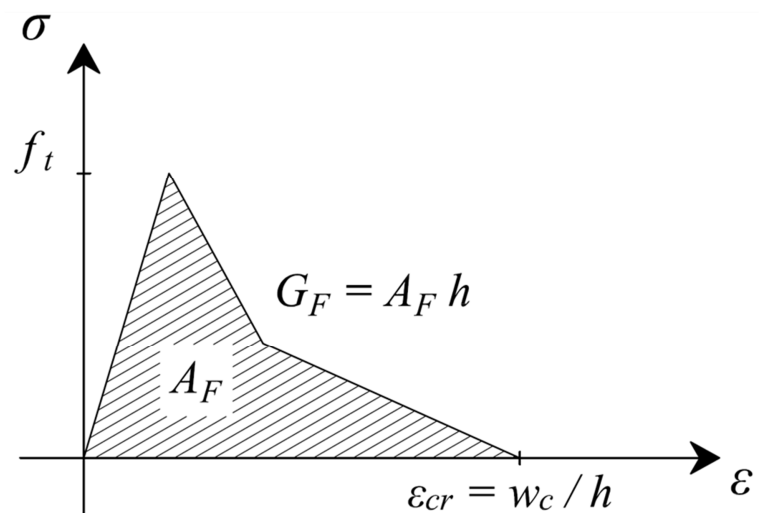

Figure 2: Bi-linear stress-strain softening law [2].

Note that $f_{\mathrm{t}}$ and $E_{\mathrm{c}}$ are tensile strength and Young's modulus of concrete, respectively. Strain $\varepsilon_{\text {cr }}$ corresponds to critical crack opening. It is calculated as $\varepsilon_{\mathrm{cr}}=w_{\mathrm{cr}} / h$ with $w_{\mathrm{cr}}=$ critical 
crack opening and $h=$ size of the finite element. In the here employed model $\psi$ is chosen based on the tensile softening curves proposed by Hoover and Bažant. [2].

Based on the micro-macro work conjugacy of volumetric-deviatoric split and using in advance defined microplane stress-strain constitutive laws, the macroscopic stress tensor is calculated as an integral over all predefined microplane orientations:

$$
\begin{aligned}
\sigma_{i j}= & \sigma_{V} \delta_{i j}+ \\
& +\frac{3}{2 \pi} \int_{S}\left(\begin{array}{c}
\sigma_{D}\left(n_{i} n_{j}-\frac{\delta_{i j}}{3}\right)+ \\
\frac{\sigma_{K}}{2}\left(k_{i} n_{j}+k_{j} n_{i}\right)+ \\
\frac{\sigma_{M}}{2}\left(m_{i} n_{j}+m_{j} n_{i}\right)
\end{array}\right) d S
\end{aligned}
$$

in which $S$ denotes the surface of the unit radius sphere and $\delta_{i j}$ is Kronecker delta. To account for large strains and large displacements, Green-Lagrange finite strain tensor is used together with co-rotational Cauchy stress tensor.

The rate dependency in the here used version of the microplane model for concrete accounts for two effects: (1) the rate dependency related to the formation (propagation) of the micro-cracks, which is the effect of inertia at the level of the micro-crack tip, and (2) the rate dependency due to the viscosity of concrete (bulk material) between the micro-cracks. The rate effect on each microplane component is defined according to rate process theory. Consequently, the rate dependency for each microplane component reads $[3,4]$ :

$$
\begin{aligned}
& \sigma_{\mathrm{m}}\left(\varepsilon_{\mathrm{m}, \mathrm{ef}}\right)= \\
& =\sigma_{\mathrm{m}}^{0}\left(\varepsilon_{\mathrm{m}, \mathrm{ef}}\right)\left[1+c_{2} \operatorname{asinh}\left(\frac{\dot{\gamma}}{c_{1}}\right)\right] \\
& \text { with } \quad \dot{\gamma}=\sqrt{\frac{1}{2} \dot{\varepsilon}_{i j} \dot{\varepsilon}_{i j}} \quad c_{1}=\frac{c_{0}}{s_{\mathrm{cr}}}
\end{aligned}
$$

where $c_{0}$ and $c_{2}$ are material rate constants, which have to be calibrated by fitting test data, $s_{\mathrm{cr}}$ is assumed spacing of micro-cracks and $\dot{\varepsilon}_{i j}$ are components of the macroscopic strain rate tensor. From Eq. (3) is obvious that the rate magnitude is not measured on the individual microplane, which would be not objective, but on the macro scale. Furthermore, Eq. (3) applies to all microplane components except to volumetric compression, which is assumed to be rate insensitive. This is because for volumetric compression there is no crack development, i.e. the material is compacted. The model parameters from Eq. (3) are calibrated based on the uni-axial compressive tests performed by [5]. For more detail see [3].

\section{MESH SENSITIVITY STUDY}

In order to investigate whether the crack band regularization scheme is able to realistically predict the response of concrete for different sizes of finite elements mesh sensitivity study on CT specimen was carried out. In the study two different types of standard solid finite elements are used, eightnode linear strain elements and four-node constant strain elements. The analysis is carried out for the CT specimen, which was recently experimentally tested for different loading rates [6]. The geometry of the specimen and the test set-up is shown Fig. 3. The concrete properties are summarized in Table 1.

Table 1: Material properties

\begin{tabular}{lc}
\hline \multicolumn{2}{c}{ Concrete properties (quasi-static) } \\
\hline \hline Density [kg/m $\left.{ }^{3}\right]$ & 2400 \\
Elastic modulus [GPa] & 36.0 \\
Poisson's ratio & 0.18 \\
Uniaxial compr. strength $[\mathrm{MPa}]$ & 53 \\
Tensile strength [MPa] & 3.8 \\
Fracture energy [J/m $\left.{ }^{2}\right]$ & 65 \\
\hline
\end{tabular}

In the experimental investigations, CT specimen was loaded over the steel frame with a loading range up to $8 \mathrm{~m} / \mathrm{s}$ (Fig. 3). It was found that the resistance increases with the increase of loading rate. For loading rates up to approximately $3 \mathrm{~m} / \mathrm{s}$ the increase of resistance was linear in semi log scale.

In this loading range only a single crack was observed. Increasing the loading rate, the crack tends to be more inclined with respect to 
the loading direction. However, for loading rates above $3.0 \mathrm{~m} / \mathrm{s}$, the increase of resistance was progressive and also crack branching was observed. Maximum crack velocity of approximately $800 \mathrm{~m} / \mathrm{s}$ was measured.
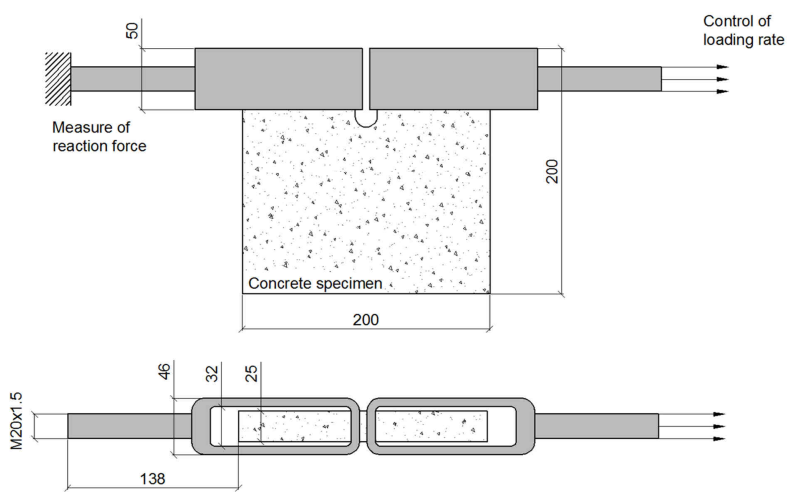

Figure 3: Geometry, load and boundary conditions of the CTS (mm).

Typical experimental crack patterns for quasi-static and dynamic loading are shown in Fig. 4. More details related to the experimental tests can be found in [6].
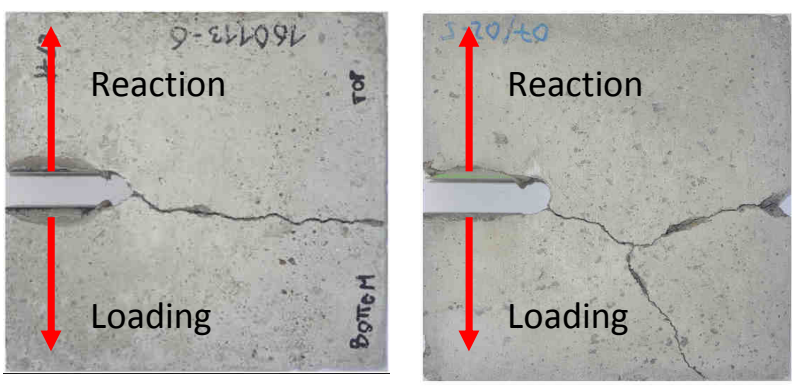

Figure 4: Typical experimentally obtained crack patterns for loading rates of $0.035 \mathrm{~m} / \mathrm{s}$ (left) and $3.3 \mathrm{~m} / \mathrm{s}$ (right).

In numerical pre- and post-test numerical studies [3, 7] the finite element analysis was carried out using explicit finite element code based on the rate sensitive microplane model. To avoid mesh sensitivity crack band method was used. Although it was shown that for dynamic analysis of CT specimen crack band method assures objective results [7], no detailed mesh sensitivity study was carried out. Therefore, to validate the mesh independency when using local crack band approach, for the tested CT specimen mesh sensitivity study is carried out.

In the analysis the same geometry and boundary conditions (Fig. 3), as well as the same concrete properties are used (Tab. 1). The typical discretization of the test set-up is shown in Fig. 5.

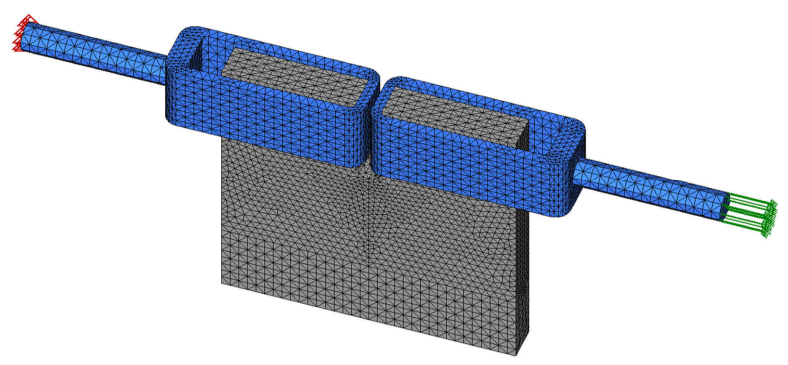

Figure 5: Typical discretization of the test set-up, $h=5$ mm 4-nodes solids.

The typical curves for uniaxial stress-strain response of concrete $(h=15 \mathrm{~mm})$ are plotted in Fig. 6.

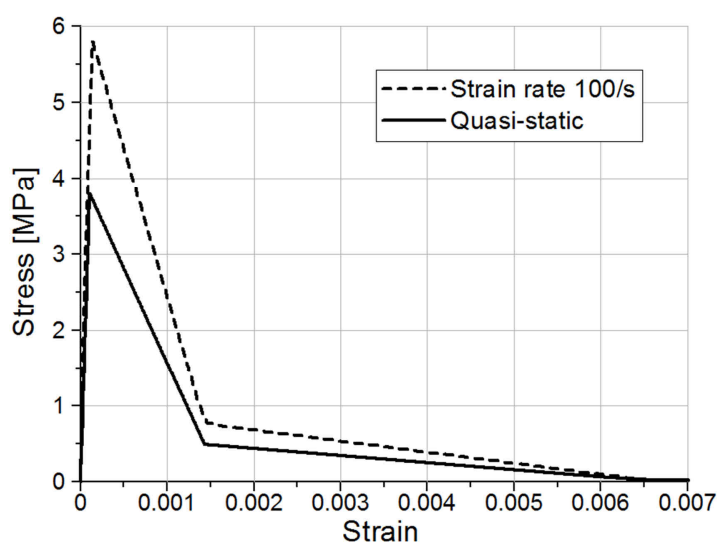

Figure 6: Typical stress strain response of concrete ( $h=15 \mathrm{~mm}$ ) for quasi-static and dynamic loading $(\dot{\varepsilon}=100 / \mathrm{s})$.

As can be seen for tension softening, bi-linear response is adopted, following the proposal from [2]. Plotted are curves for quasi-static loading and for dynamic loading (strain rate $\dot{\varepsilon}=100 / \mathrm{s}$ ). Note that rate dependency is accounted for according to Eq. (3).

The analysis is performed for three different FE discretizations (Fig. 7): fine, medium and coarse, with element sizes of $h=5,10$ and 15 $\mathrm{mm}$, respectively. Two different standard finite elements are used, four- and eight-node solids. The mesh sensitivity study is performed for quasi static and dynamic loading with displacement loading rate of $3.30 \mathrm{~m} / \mathrm{s}$. As in the experiment, the load was performed by 
displacement control (see Fig. 3).

(a)

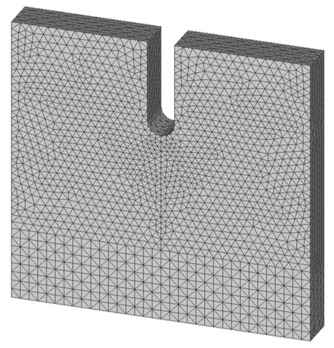

(b)

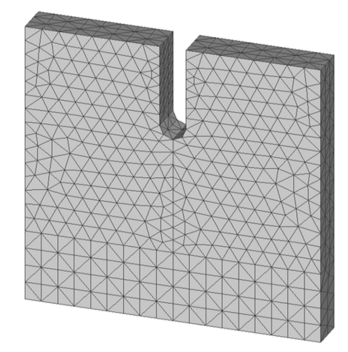

(c)
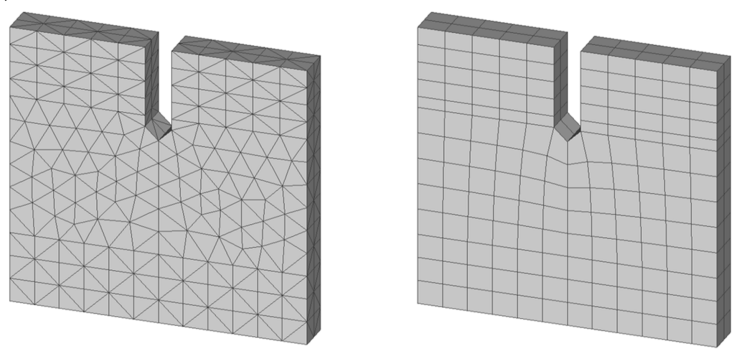

Figure 7: Spatial discretizations of CT specimen, fournode solid FE (left) and eight-node FE (right): (a) $h=5$ $\mathrm{mm}$, (b) $h=10 \mathrm{~mm}$ and (c) $h=15 \mathrm{~mm}$.

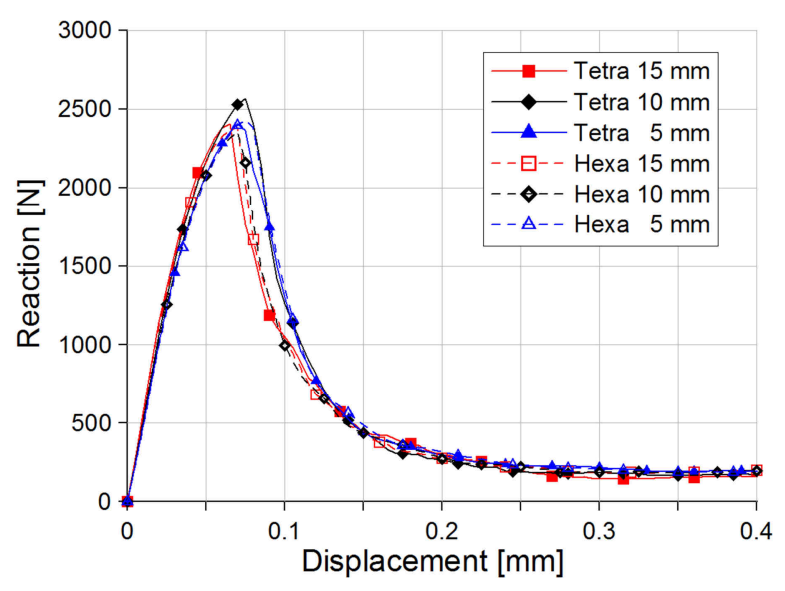

Figure 8: Reaction-displacement curves for all discretizations (quasi-static analysis).

Fig. 8 shows computed reactiondisplacement curves for quasi-static loading and the peak loads are summarized in Tab. 2 . As can be seen, although the meshes and finite elements are quite different there is no significant influence of the discretization on the response. The corresponding crack patterns for fine and coarse mesh (four-node solids) are shown in Fig. 9. As expected, crack propagates perpendicular to the loading direction.

Table 2: Summary of peak resistance

\begin{tabular}{ccc}
\hline $\begin{array}{c}\text { Element size } \\
(\mathrm{mm})\end{array}$ & $\begin{array}{c}\text { Element } \\
\text { type }\end{array}$ & $\begin{array}{c}\text { Peak } \\
\text { Reaction }(\mathrm{N})\end{array}$ \\
\hline \hline 5 & 4-nodes & 2406.5 \\
5 & 8-nodes & 2430.6 \\
10 & 4-nodes & 2566.1 \\
10 & 8-nodes & 2353.4 \\
15 & 4-nodes & 2404.0 \\
15 & 8-nodes & 2382.3 \\
\hline
\end{tabular}

(a)

(b)
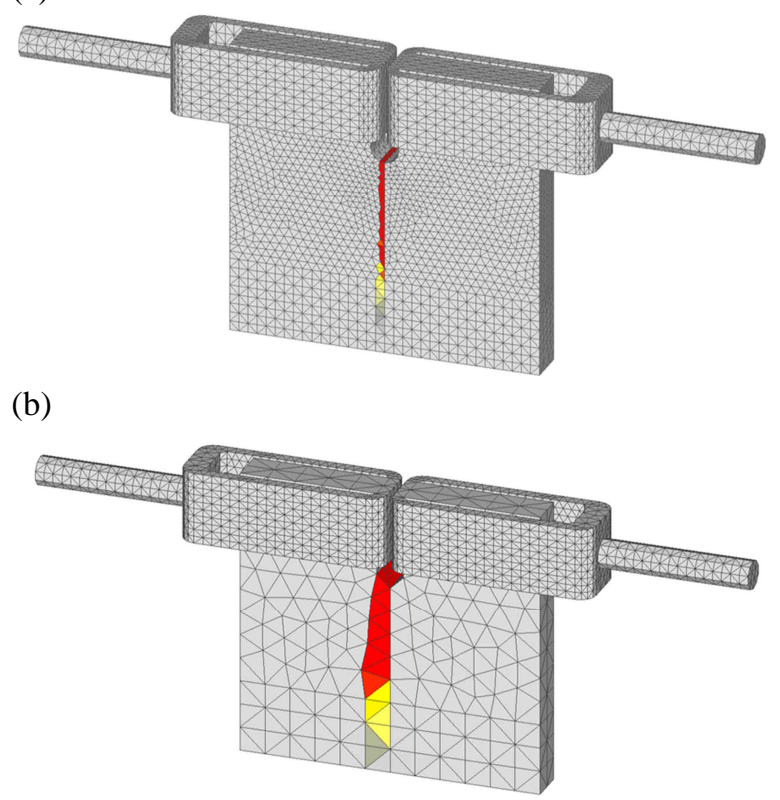

Figure 9: Predicted crack patterns for quasi static loading (max. principal strains, red = critical crack opening of $0.10 \mathrm{~mm}$ ), 4-node solids: (a) $h=5 \mathrm{~mm}$ and (b) $h=15 \mathrm{~mm}$.

Furthermore, the influence of the mesh size is investigated for dynamic loading with loading rate of $3.30 \mathrm{~m} / \mathrm{s}$ applied at the end of the steel loading frame (Fig. 5). The resulting reaction histories are shown in Fig. 10. As expected, the reaction peak is in this case much higher than in the static analysis. As discussed in detail in $[2,7]$ this is a consequence of rate sensitivity and inertia effects. At higher strain rates, larger than approximately 100/s, the effects of inertia are 
responsible for progressive increase of resistance and for crack branching [2]. Similar as for quasi-static analysis, reaction history response is not much dependent on the discretization. Moreover, the agreement with experimental results is also reasonably good.

(a)

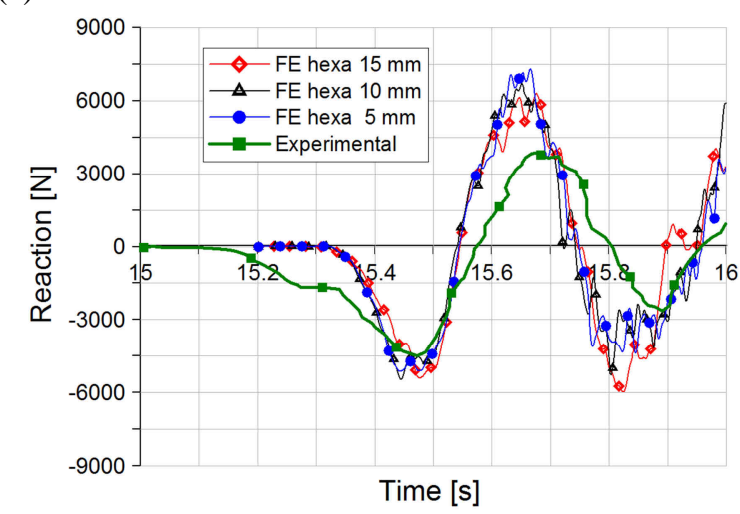

(b)

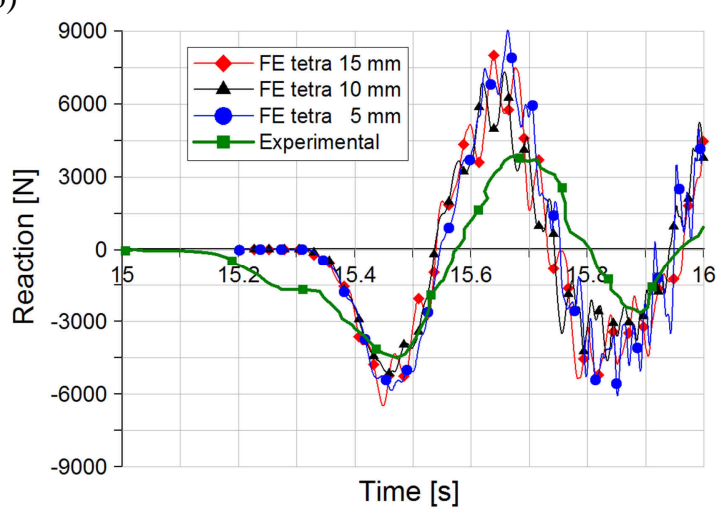

Figure 10: Experimental and numerically predicted reaction - time response for loading rate of $3.30 \mathrm{~m} / \mathrm{s}$ : (a)

8-nodes elements, (b) 4-nodes elements.

The predicted crack patterns for all discretizations are shown in Fig. 11. As can be seen, although the meshes are quite different, they are very similar. It is important to note that even for relatively coarse meshes crack branching is reasonably well predicted. Furthermore, all crack patters are in good agreement with the experimental crack pattern (see Fig. 4, right).

In Fig. 12 are plotted crack patterns with the corresponding crack velocities. They are all rather similar and agree well with the experimentally measured values [2]. Maximum crack speed of approximately 800 $\mathrm{m} / \mathrm{s}$ is obtained before crack branching. (a)
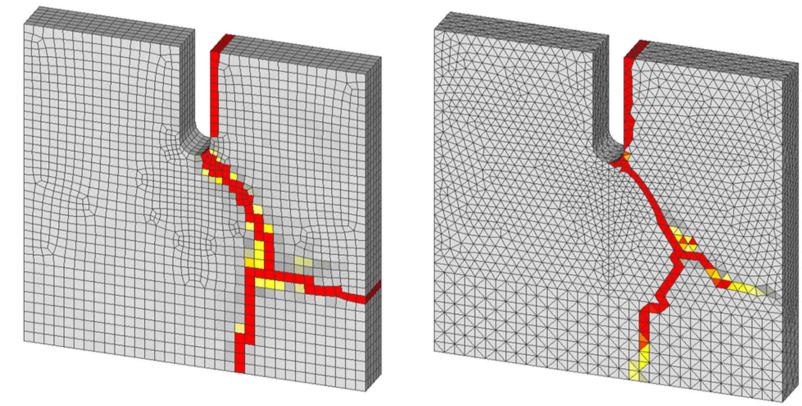

(b)
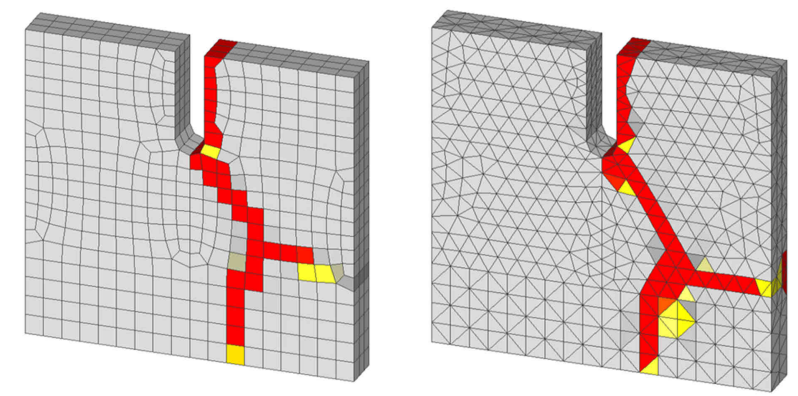

(c)
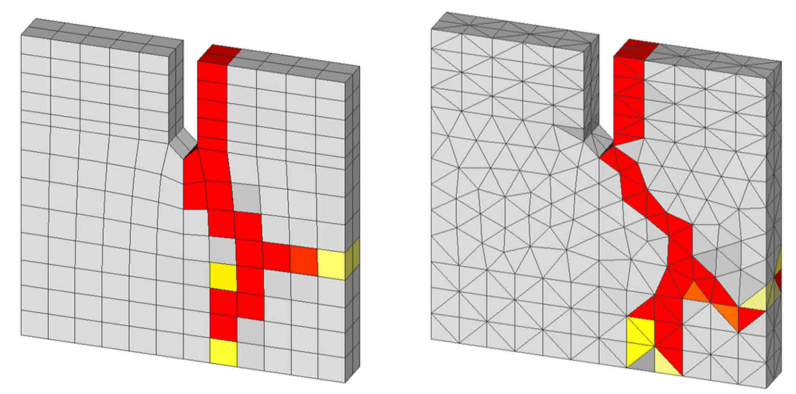

Figure 11: Predicted crack patterns for dynamic load, left 8-node solids and right 4-node solids for: (a) $h=5$ $\mathrm{mm}$, (b) $h=10 \mathrm{~mm}$ and (c) $h=15 \mathrm{~mm}$.

\section{CONCLUSIONS}

In the present paper mesh sensitivity study for concrete CT specimen loaded under quasi static and dynamic conditions is carried out. Based on the results of the study the following conclusions can be drawn out.

(1) The employed rate sensitive microplane model based on the bilinear stress-strain (crack opening) law is able to realistically predict resistance and crack pattern for quasi-static and dynamic loading.

(2) It is shown that simple local finite element analysis based on the energy dissipation, which should be independent of the size of the finite elements (crack band method), does not exhibit mesh sensitivity. 
(a)

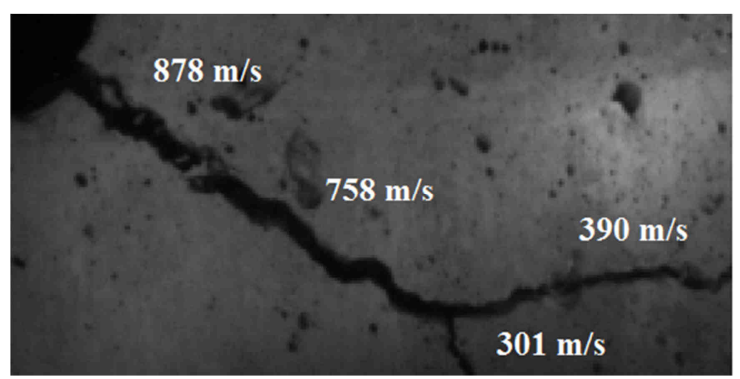

(b)
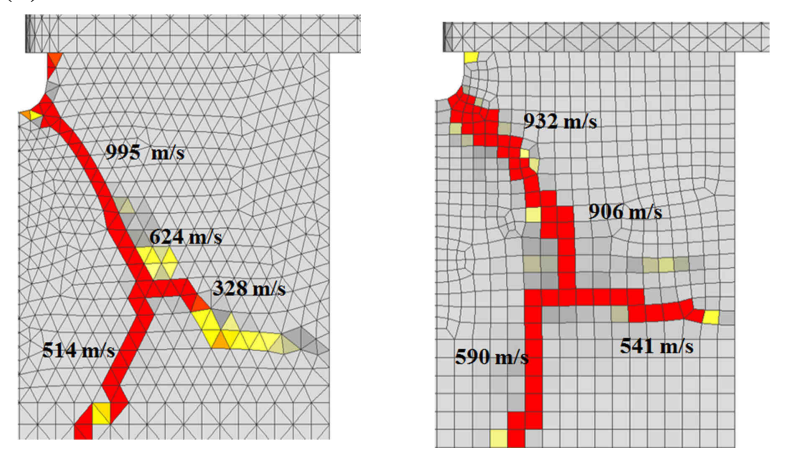

(c)

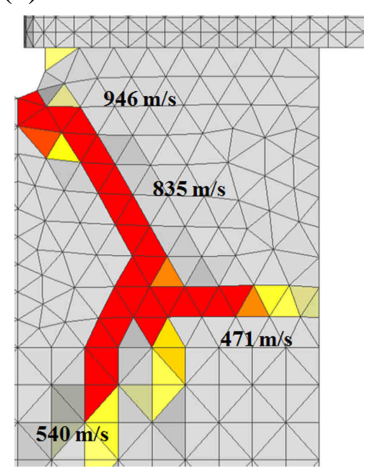

(d)
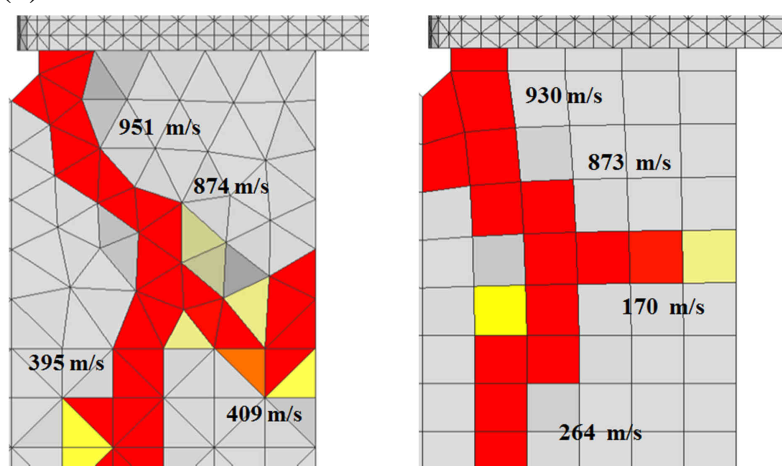

Figure 12: Experimentally measured crack speed (a) and predicted, left 8-node solids and right 4-node solids for: (a) $h=5 \mathrm{~mm}$, (b) $h=10 \mathrm{~mm}$ and (c) $h=15 \mathrm{~mm}$.

(3) Although the investigated discretizations are rather different, for dynamic loading crack patterns, crack branching and crack velocities are very similar and all agree well with the experimental tests.
(4) For the investigated CT specimen the failure mode corresponds to mode-I fracture type. Obviously for this type of failure mode crack band method is sufficiently accurate with respect to the size and type of finite elements. However, dynamic fracture for more complex failure modes, such as compressionshear, should be studied as well.

\section{REFERENCES}

[1] Ožbolt J., Li Y., and Kožar I. 2001. Microplane model for concrete with relaxed kinematic constraint. International Journal of Solids and Structures 38.16: 2683-2711.

[2] Hoover C. G., and Bažant Z. P. 2014. Cohesive crack, size effect, crack band and work-of-fracture models compared to comprehensive concrete fracture tests. International Journal of Fracture 187(1): 133-143.

[3] Ožbolt J., Rah K. K., and Meštrović D. 2006. Influence of loading rate on concrete cone failure. International Journal of Fracture 139.2: 239-252.

[4] Bažant Z. P. et al. 2000. Fracturing rate effect and creep in microplane model for dynamics. Journal of Engineering Mechanics 126.9: 962-970.

[5] Dilger W. H., Koch R., and Kowalczyk R. 1984. Ductility of plain and confined concrete under different strain rates. Journal Proceedings. Vol. 81. No. 1.

[6] Ožbolt J., Bošnjak J., Sola E. 2013. Dynamic fracture of concrete compact tension specimen: Experimental and numerical study. Int J Solids Struct 50: 4270-4278.

[7] Ožbolt J., Sharma A., and Reinhardt H.W. 2011. Dynamic fracture of concretecompact tension specimen. International journal of solids and structures 48.10: 1534-1543. 